\title{
Quantum reflection of antihydrogen from the Casimir potential above matter slabs
}

\author{
G. Dufour, ${ }^{1}$ A. Gérardin, ${ }^{1}$ R. Guérout, ${ }^{1}$ A. Lambrecht, ${ }^{1}$ V.V. Nesvizhevsky, ${ }^{2}$ S. Reynaud, ${ }^{1}$ and A.Yu. Voronin ${ }^{3}$ \\ ${ }^{1}$ Laboratoire Kastler-Brossel, CNRS, ENS, UPMC, Campus Jussieu, F-75252 Paris, France \\ ${ }^{2}$ Institut Laue-Langevin (ILL), 6 rue Jules Horowitz, F-38042, Grenoble, France \\ ${ }^{3}$ P.N. Lebedev Physical Institute, 53 Leninsky prospect, Ru-117924 Moscow, Russia
}

\begin{abstract}
We study quantum reflection of antihydrogen atoms from matter slabs due to the van der Waals/Casimir-Polder (vdW/CP) potential. By taking into account the specificities of antihydrogen and the optical properties and width of the slabs we calculate realistic estimates for the potential and quantum reflection amplitudes. Next we discuss the paradoxical result of larger reflection coefficients estimated for weaker potentials in terms of the Schwarzian derivative. We analyze the limiting case of reflections at small energies, which are characterized by a scattering length and have interesting applications for trapping and guiding antihydrogen using material walls.
\end{abstract}

PACS numbers: $36.10 . \mathrm{Gv}, 34.35 .+\mathrm{a}, 12.20 \mathrm{Ds}$

\section{INTRODUCTION}

Quantum reflection is the process of reflecting particles from an attractive but rapidly varying potential. It has been studied since the early days of quantum theory [1. On the other hand it is well known that atoms in the vicinity of a surface experience the long-range van der Waals/Casimir-Polder potential (vdW/CP) [2]. Quantum reflection occurs here if the atom enters a region where the potential varies rapidly compared with the atom's wavelength. Experimentally quantum reflection on the vdW/CP potential has been observed with slow atoms reflected from a liquid Helium surface [35] or from solid surfaces [6, 7]. More recent efforts have focused on quantum reflection from rough or micro/nanostructured surfaces 8 10 and on quantum reflection of Bose-Einstein condensates on flat or nanostructured silicon [11, 12].

The theoretical description of quantum reflection has been the topic of numerous contributions in the past 1318, which are presented in some detail in [19. The particular case of reflection on a vdW/CP potential created by thin slabs or graphene sheets has been studied recently in 20. In addition it has been put forward that quantum reflection coefficients can be tuned using external optical fields [21] or via thermal non-equilibrium effects [22].

In the present paper, we will study the quantum reflection of antihydrogen atoms $\overline{\mathrm{H}}$ falling on material walls. As $\overline{\mathrm{H}}$ atoms are annihilated in contact with matter, this case enforces specific boundary conditions at the material surface 23. In particular the behavior of the short-range atom-wall potential becomes irrelevant as all antiatoms that come close enough to the surface are annihilated. This topic is important to the GBAR collaboration which aims to measure the gravitational behavior of $\overline{\mathrm{H}}$ by studying its time of free fall from a well-defined trap to a matter plate 24]. We will give accurate estimations for the van der Waals/Casimir-Polder (vdW/CP) potential between the antiatoms and the surface as well as for the associated quantum reflection.

A number of different methods are available to calcu- late atom-surface dispersion forces 25 28, (see 29] for a detailed bibliography). Here we will use the scattering approach [30, 31] which has been developed to calculate Casimir forces in arbitrary geometries and which can be applied to the study of vdW/CP forces between an atom and flat or nanostructured surfaces $[32$. In order to obtain accurate estimations, it will in particular be necessary to take into account the material properties and the finite thickness of the slabs 33 .

In order to explain the paradoxical result that larger reflections are obtained for weaker potentials, we will discuss how the quantum reflection occurs when the atoms approach the surface and draw a relation to the Schwarzian derivative. We will finally analyze the limiting case of reflections at small energies, which have interesting applications for trapping and guiding antihydrogen with material walls [34, 35. Quantum reflection is characterized by a scattering length which we will calculate for different materials and different slab widths. We note at this point that quantum reflection is calculated in the present paper from a static potential, so that the role of dissipation in matter is neglected [36].

\section{CASIMIR-POLDER POTENTIAL}

We use the scattering formalism 30, 31 applied here to the Casimir-Polder potential between an atom and a plate :

$$
V(z)=\hbar \int_{0}^{\infty} \frac{\mathrm{d} \xi}{2 \pi} \operatorname{Tr} \ln \left(1-\mathcal{R}_{\mathrm{P}} e^{-\kappa z} \mathcal{R}_{\mathrm{A}} e^{-\kappa z}\right)
$$

As the quantum reflection process is expected to occur at distances smaller than $1 \mu \mathrm{m}$ (more discussions below), and thus smaller than the typical thermal wavelength, this formula has been written at zero temperature. The matrices $\mathcal{R}_{\mathrm{P}}$ and $\mathcal{R}_{\mathrm{A}}$ describe the reflection of the electromagnetic vacuum fields on the plate and atom respectively. They are calculated for a Wick rotated complex frequency $\omega=i \xi$ with the trace (Tr) bearing on transverse wave vectors $\mathbf{k}$ and polarizations $p=\mathrm{TE}, \mathrm{TM}$. The 
factor $e^{-\kappa z}$ accounts for propagation between the atom and plate where $\kappa=\sqrt{\mathbf{k}^{2}+\xi^{2} / c^{2}}$ is the Wick rotated complex longitudinal wavevector.

We may safely neglect all multiple reflections between the atom and the surface and thus expand the general scattering formula (1) to first order in $\mathcal{R}_{\mathrm{A}}$. When the scattering on the atom is described in the dipolar approximation [32, the potential is read in terms of a dynamic atomic polarizability $\alpha$, given in units of a volume:

$$
\begin{aligned}
V(z)= & \frac{\hbar}{c^{2}} \int_{0}^{\infty} \mathrm{d} \xi \xi^{2} \alpha(i \xi) \int \frac{\mathrm{d}^{2} \mathbf{k}}{(2 \pi)^{2}} \frac{e^{-2 \kappa z}}{\kappa} \\
& \times\left[\rho^{\mathrm{TE}}-\left(1+\frac{2 c^{2} k^{2}}{\xi^{2}}\right) \rho^{\mathrm{TM}}\right] .
\end{aligned}
$$

The $\rho^{p}$ denote the electromagnetic reflection amplitudes for the two polarizations $p=$ TE, TM. We study first the case of reflection from a semi-infinite bulk, described by the Fresnel laws expressing continuity relations at the interface :

$$
\rho_{\text {bulk }}^{\mathrm{TE}}=\frac{\kappa-K}{\kappa+K} \quad, \quad \rho_{\text {bulk }}^{\mathrm{TM}}=\frac{\epsilon(i \xi) \kappa-K}{\epsilon(i \xi) \kappa+K},
$$

where $K=\sqrt{\mathbf{k}^{2}+\epsilon(i \xi)(\xi / c)^{2}}$ corresponds to the Wick rotated longitudinal wavevector inside the medium, and $\epsilon$ is the relative dielectric function of this medium (evaluated at the Wick rotated complex frequency).

The results presented below use the following optical response properties :

1. The atomic polarizability is that of antihydrogen $(\overline{\mathrm{H}})$, and is assumed to be the same as that of hydrogen $(\mathrm{H})$ [37].

2. Perfect mirrors have been used in previous calculations 18, 23, 37.

$$
\rho^{\mathrm{TE}} \equiv-1 \quad, \quad \rho^{\mathrm{TM}} \equiv 1 ;
$$

they are considered here for the sake of comparison with results obtained with the real materials discussed below.

3. Mirrors made of intrinsic silicon are described by a Drude-Lorentz model [32, 33] :

$$
\epsilon(i \xi)=\epsilon_{\infty}+\frac{\left(\epsilon_{0}-\epsilon_{\infty}\right) \omega_{0}^{2}}{\xi^{2}+\omega_{0}^{2}}
$$

with the parameters $\epsilon_{0}=11.87, \epsilon_{\infty}=1.035$, $\omega_{0}=6.6 \times 10^{15} \mathrm{rad} . \mathrm{s}^{-1}$.

4. Mirrors made of amorphous silica are described by a simple Sellmeier model 38] :

$$
\epsilon(i \xi)=1+\sum_{i=1,2,3} \frac{B_{i}}{1+\left(\xi / \omega_{i}\right)^{2}},
$$

with the parameters $B_{1,2,3}=0.696749,0.408218$, 0.890815 and $\omega_{1,2,3}=27.2732,16.2858,0.190257$ $\times 10^{15} \mathrm{rad} . \mathrm{s}^{-1}$.
5. The electronic properties of graphene are described by a Dirac model leading to reflection coefficients given in 39 .

The potential (2) has well-known asymptotic behaviors at short and long distances

$$
V(z) \underset{z \ll \ell}{\rightarrow}-\frac{C_{3}}{z^{3}} \quad, \quad V(z) \underset{z \gg \ell}{\rightarrow}-\frac{C_{4}}{z^{4}} .
$$

where $\ell$ is a distance scale determined by the characteristic atomic frequencies which enter the expressions of polarizability or dielectric function. The short distance limit is identical to the famous London/Van der Waals result while the long distance limit is the so-called retarded Casimir-Polder interaction which takes into account that the finite speed of light comes into play at large separations 2. The values given in Table I are obtained from the exact vdW/CP potential (2) and given in atomic units.

\begin{tabular}{|c|c|c|c|}
\hline & perfect & silicon & silica \\
\hline$C_{3}$ & 0.25 & 0.10 & 0.05 \\
\hline$C_{4}$ & 73.6 & 50.3 & 28.1 \\
\hline
\end{tabular}

TABLE I. Coefficients $C_{3}$ and $C_{4}$ for the vdW/CP interaction for $\overline{\mathrm{H}}$ atoms above perfect mirrors, silicon and silica bulks ; the values are given in atomic units $\mathrm{E}_{\mathrm{h}} \mathrm{a}_{0}^{n}$ for $C_{n}$ (Hartree energy $\mathrm{E}_{\mathrm{h}} \simeq 4.3597 \mathrm{aJ} ;$ Bohr radius $\mathrm{a}_{0} \simeq 52.917 \mathrm{pm}$ ).

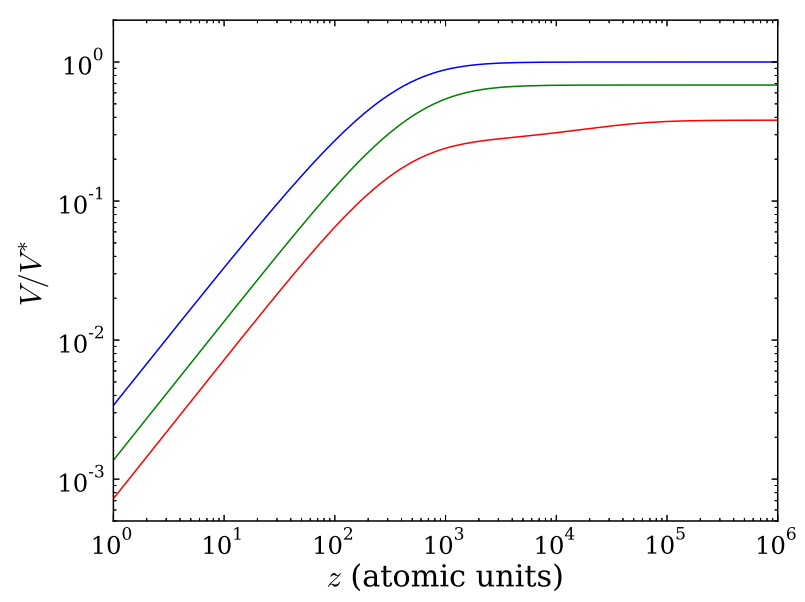

FIG. 1. (Color online) Casimir-Polder potential for $\overline{\mathrm{H}}$ in the vicinity of a material bulk, drawn as a ratio $V / V^{*}$ to the retarded potential $V^{*}$ for a perfect mirror ; from top to bottom, perfect mirror (blue), silicon (green), silica (red).

Figure 1 displays the exact vdW/CP potentials obtained from (2) for $\overline{\mathrm{H}}$ atoms on perfect mirrors and bulk mirrors made of intrinsic silicon or amorphous silica, described by eqs. (4), (5) and (6) respectively. All cases are drawn as ratios $V(z) / V^{*}(z)$ to the retarded CP limit calculated for a perfectly reflecting wall $V^{*}=-C_{4}^{*} / z^{4}$ with 
$C_{4}^{*}=2.5 \cdot 10^{-57} \mathrm{~J} \mathrm{~m}^{4}$ (=73.6a.u., see Table I). The ratios tend to constant values $C_{4} / C_{4}^{*}$ at large distances and linear variations $C_{3} z / C_{4}^{*}$ at small distances. Of course, lesser and lesser reflective materials produce weaker and weaker CP potentials, from perfect mirrors to silicon and silica plates.

\section{QUANTUM REFLECTION OF $\overline{\mathrm{H}}$}

We will now solve the problem of quantum reflection of $\overline{\mathrm{H}}$ atoms from the $\mathrm{CP}$ potential calculated in the previous section, starting from free atoms with an energy $E>0$ just before they feel the CP potential. We will also use below the notation $h$ for the height of free fall of the atoms with the correspondence $E=m g h$ (supposing $h$ much larger than tens of microns).

The Schrödinger equation may be written :

$$
\psi^{\prime \prime}(z)+\frac{p^{2}(z)}{\hbar^{2}} \psi=0,
$$

where primes denote derivations with respect to $z$ while $p^{2}$ is the square of the semiclassical momentum

$$
p(z)=\sqrt{2 m(E-V(z))} .
$$

The general solution can be expressed, without approximation, as a superposition of the two WKB waves

$$
\psi(z)=\frac{c_{+}(z)}{\sqrt{|p(z)|}} e^{i \phi(z)}+\frac{c_{-}(z)}{\sqrt{|p(z)|}} e^{-i \phi(z)},
$$

where $\phi$ is the WKB phase $\left(z_{0}\right.$ arbitrary)

$$
\phi(z)=\int_{z_{0}}^{z} \frac{p\left(z^{\prime}\right) \mathrm{d} z^{\prime}}{\hbar} .
$$

The Schrödinger equation (8) is obeyed when the amplitudes $c_{ \pm}$verify the coupled first-order equations [13]

$$
c_{ \pm}^{\prime}(z)=e^{\mp 2 i \phi(z)} \frac{p^{\prime}(z)}{2 p(z)} c_{\mp}(z) .
$$

As $\overline{\mathrm{H}}$ annihilates as soon as it touches the wall, there cannot be any wave reflected immediately from the surface $z=0$ of the material boundary 23. This full absorption condition imposes $c_{+}(z=0)=0$ and we are then free to fix $c_{-}(z=0)=1$. The quantum reflection amplitude $r$ is thus given by the ratio of the amplitudes $c_{+}(z)$ and $c_{-}(z)$ at the limit $z \rightarrow \infty$ (see equation 10 . Finally, the quantum reflection probability discussed below is the squared modulus of this amplitude $|r|^{2}$.

In order to numerically integrate the preceding equations, it remains to fix the problems arising from the divergence of the potential in the vicinity of the surface. It will result from forthcoming discussions that the WKB waves are well defined near the wall. However a difficulty arises from the divergence of the WKB phase $\phi$ there. To fix this difficulty, we proceed as in 23] by studying the analytical form of the solution for $c_{ \pm}$close enough to the wall. The potential there takes its van der Waals approximated form while the energy $E$ becomes negligible when compared to the potential.

In this limit the functions $f_{ \pm}(t)$ defined by $c_{ \pm}(z)=x^{3 / 2} f_{ \pm}(t), x=\sqrt{8 m C_{3} / z}$ and $t= \pm 2 i x$ satisfy the Kummer equation:

$$
t f_{ \pm}^{\prime \prime}(t)+(b-t) f_{ \pm}^{\prime}(t)-a f_{ \pm}(t)=0
$$

with parameters $a=3 / 2$ and $b=4$. A pair of independent solutions is given by Kummer's confluent hypergeometric functions $M(a, b, t)$ and $U(a, b, t)$ 40. On the other hand the Schrödinger equation (8) can also be solved close to the wall. The two counterpropagating waves can be expressed in terms of the Hankel functions as $H_{1}^{(1)}(x) / x$ and $H_{1}^{(2)}(x) / x$ and the full absorption condition imposes that the second wave has a null amplitude 23. By comparing this expression of the wave function with 10 we find that

$$
\begin{aligned}
c_{+}(x)=-2(1+i) x^{3 / 2} & {\left[U\left(\frac{3}{2}, 4,2 i x\right)\right.} \\
- & \left.\frac{i \sqrt{\pi}}{8} M\left(\frac{3}{2}, 4,2 i x\right)\right] e^{-2 i x_{0}} \\
c_{-}(x) & =-2(1+i) x^{3 / 2} U\left(\frac{3}{2}, 4,-2 i x\right)
\end{aligned}
$$

A better behavior of the functions is thus obtained by changing the variables $z \rightarrow x$ in the vicinity of matter and matching the numerical solutions to the known analytical solutions (14). The results shown below are obtained in this manner close to the wall, while variables are switched back to $z$ when the potential becomes comparable to the energy.

We show in figure 2 the numerical solution for quantum reflection probability obtained with the exact $\mathrm{CP} / \mathrm{vdW}$ potentials discussed in the preceding section for $\overline{\mathrm{H}}$ atoms falling to perfect mirrors and bulk mirrors made of silicon or silica. It turns out that significant values are obtained for the quantum reflection probability with the typical numbers considered for the project GBAR as shown in Table 【.

\begin{tabular}{|c|c|c|c|}
\hline & perfect & silicon & silica \\
\hline$|r|^{2}$ & $14 \%$ & $20 \%$ & $32 \%$ \\
\hline
\end{tabular}

TABLE II. Reflection probabilities for a free fall height $h \sim 10 \mathrm{~cm}$, which corresponds to an energy per unit mass $g h \sim 1(\mathrm{~m} / \mathrm{s})^{2}$ at the matter plate.

These numbers highlight a striking result of the calculations which is also emphasized by the use of the same color codes in figures 1 and 2 : when going to lesser and lesser reflective materials, i.e. weaker and weaker $\mathrm{CP} / \mathrm{vdW}$ interactions, one indeed obtains larger and larger quantum reflection probability [12, 20. This 


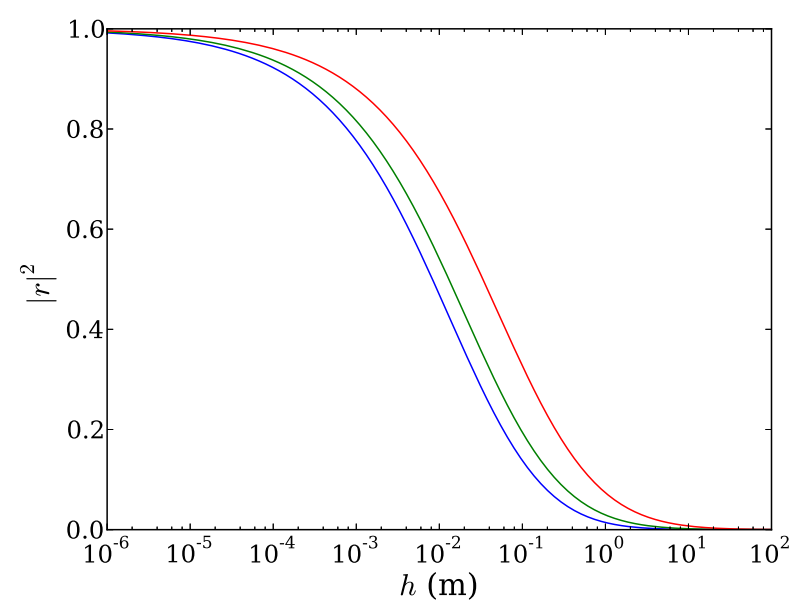

FIG. 2. (Color online) Quantum reflection probability $|r|^{2}$ as a function of the free fall height $h$ for $\overline{\mathrm{H}}$ atoms on bulk mirrors ; from bottom to top, perfect mirror (blue), silicon (green), silica (red).

apparent paradox is analyzed in the next section, by taking a closer look at the region where quantum reflection occurs.

\section{BADLANDS CONDITION}

We now discuss the so-called badlands condition for efficient reflection, that is also for significant non adiabatic transitions beyond the WKB approximation [13, 19].

To this aim, we recall that the WKB approximation $\widetilde{\psi}$, the wavefunction 10 with constant amplitudes $c_{ \pm}$, also obeys a Schrödinger's equation :

$$
\begin{aligned}
& \widetilde{\psi}^{\prime \prime}(z)+\frac{\widetilde{p}^{2}(z)}{\hbar^{2}} \widetilde{\psi}(z)=0, \\
& \widetilde{p}^{2}(z) \equiv p^{2}(z)+\frac{\hbar^{2}}{2} \mathcal{S} \phi(z) .
\end{aligned}
$$

The difference between (8) and (15) is the extra term in $\widetilde{p}^{2}$ with respect to $p^{2}$, proportional to the Schwarzian derivative of the WKB phase :

$$
\mathcal{S} \phi(z) \equiv \frac{\phi^{\prime \prime \prime}(z)}{\phi^{\prime}(z)}-\frac{3}{2}\left(\frac{\phi^{\prime \prime}(z)}{\phi^{\prime}(z)}\right)^{2} .
$$

This means that non adiabatic processes are characterized by this Schwarzian derivative, in a similar way to non adiabatic emission of photons in vacuum after reflection from moving mirrors [4, 42.

It follows that the WKB approximation is good when the second term in $\widetilde{p}^{2}$ in 15 is much smaller than the first one. It can be shown that this is the case for the problem being studied in the present paper for short as well as long distances, which means that left- and rightwards propagation are well defined in both limits. The non adiabatic processes giving rise to quantum reflection occur in the intermediate distance range, and their efficiency is significant for large values of the quantity :

$$
Q(z) \equiv \frac{\hbar^{2} \mathcal{S} \phi}{2 p^{2}}=\frac{\hbar^{2}}{2} \frac{p^{\prime \prime}(z)}{p(z)^{3}}-\frac{3 \hbar^{2}}{4}\left(\frac{p^{\prime}(z)}{p(z)^{2}}\right)^{2}
$$

The adiabatic approximation breaks down in regions where $|Q(z)| \sim 1$, which have been dubbed the badlands. Non adiabatic quantum reflection happens there, where the notions of left- and rightwards propagation are no longer well defined.

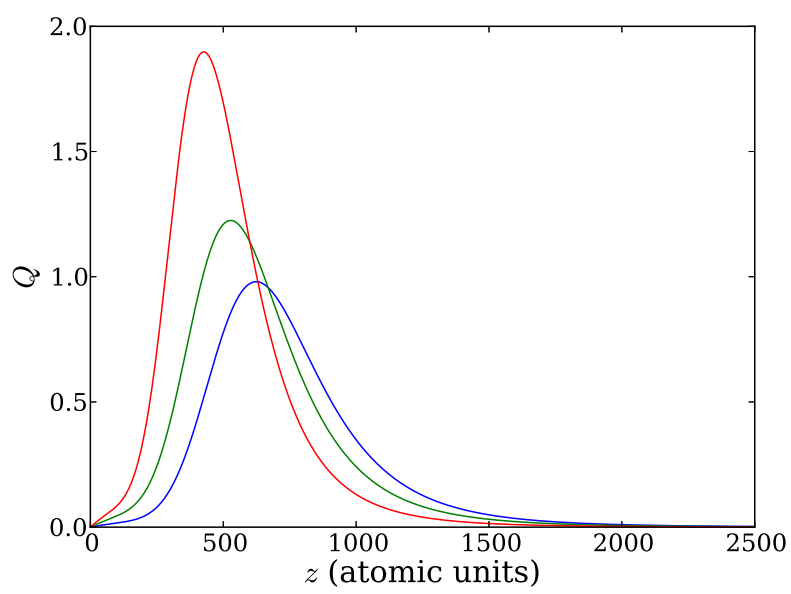

FIG. 3. (Color online) Badlands function $Q(z)$ as a function of distance to the wall (atomic units) for $\overline{\mathrm{H}}$ dropped from $h=10 \mathrm{~cm}$ on bulk mirrors ; from bottom right to top left, perfect mirror (blue), silicon (green), silica (red).

Figure 3 features the numerical evaluation of this badlands function $Q(z)$ as a function of distance $z$ to the wall (atomic units), for $\overline{\mathrm{H}}$ dropped from $h=10 \mathrm{~cm}$ on perfect, silicon or silica mirrors (same color codes as in figures 1-2). The plots clarify two features which explain the apparent paradox discussed in the preceding section. First, quantum reflection occurs closer and closer to the wall for weaker and weaker $\mathrm{CP} / \mathrm{vdW}$ interaction. Second, the value reached by $Q(z)$ is thus larger and larger, since the CP interaction gets steeper and steeper when atoms approach the wall. When considered together, these two features explain why a weaker potential leads to a more efficient reflection than a stronger one. In fact, the quantum reflection probabilities $|r|^{2}$ (see for example the numbers given in Table II increase with increasing peak value of the badlands function $Q(z)$.

\section{REFLECTION ON A THIN SLAB}

This discussion suggests that one should try to weaken further the $\mathrm{CP} / \mathrm{vdW}$ interaction with the aim of enhancing quantum reflection [20]. In the present section, we 
analyze this idea by studying either slabs having a finite thickness or a graphene layer.

The calculations proceed along the same lines as previously, except for the fact that slabs of finite thickness $d$ have smaller reflection amplitudes than the corresponding bulks. There is a general relation between these amplitudes 33] :

$$
\rho_{\mathrm{slab}}^{p}=\frac{\left(1-e^{-2 K d}\right) \rho_{\mathrm{bulk}}^{p}}{1-e^{-2 K d}\left(\rho_{\mathrm{bulk}}^{p}\right)^{2}} .
$$

When the $\mathrm{CP} / \mathrm{vdW}$ interaction is calculated at distances $z$ smaller than the thickness $d$, the results of the bulk are recovered. This can be understood from the fact that $\rho_{\text {slab }}^{p}$ goes to $\rho_{\text {bulk }}^{p}$ for large values of $d$ (up to exponentially small corrections). In contrast, the long-distance behavior of the $\mathrm{CP}$ potential is completely changed because the exponential factor now plays an important role in (18). Even the power law index is changed for the potential which now varies as :

$$
V(z) \underset{z \gg \ell, d}{\rightarrow}-\frac{C_{5}}{z^{5}}
$$

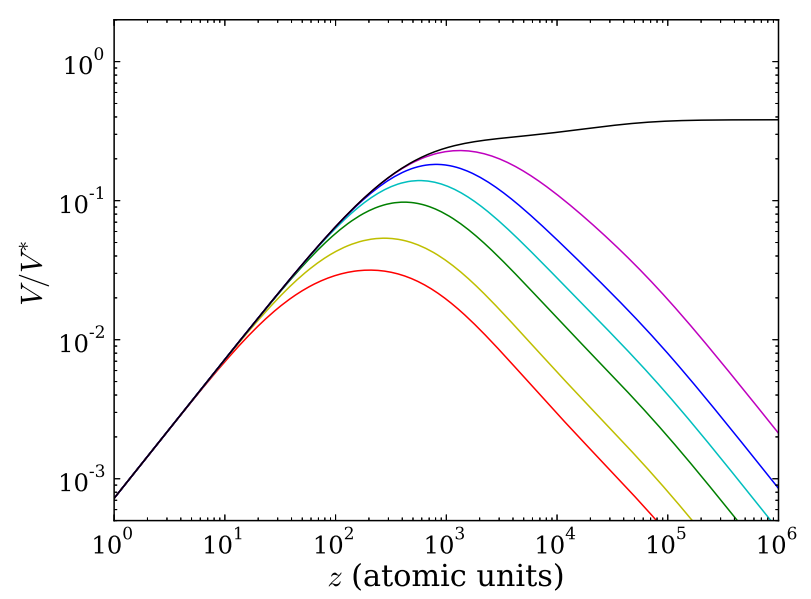

FIG. 4. (Color online) Casimir-Polder potential for $\overline{\mathrm{H}}$ in the vicinity of a silica slab, drawn as a ratio $V / V^{*}$; from top to bottom, the thickness is infinite (black), $50 \mathrm{~nm}$ (magenta), 20 $\mathrm{nm}$ (deep blue), $10 \mathrm{~nm}$ (light blue), $5 \mathrm{~nm}$ (deep green), $2 \mathrm{~nm}$ (light green) and $1 \mathrm{~nm}$ (red).

Figure 4 shows the exact $\mathrm{CP} / \mathrm{vdW}$ potentials obtained from (2) for $\overline{\mathrm{H}}$ atoms on slabs of amorphous silica, with different values for the thickness $d$. All cases are drawn as ratios of $V(z)$ to the same reference potential $V^{*}$ already used in figure 1 . The ratios tend to the same linear variations $C_{3} z / C_{4}^{*}$ at small distances as for the silica bulk (red curve in figure 1) and to inverse distance laws $C_{5} /\left(C_{4}^{*} z\right)$ at large distances with the value of $C_{5}$ being proportional to $d$. This behavior can be expected from a simple argument where the potential $V_{\text {slab }}(z)$ at distance $z$ from a slab of thickness $d$ is obtained from the difference $V_{\text {bulk }}(z)-V_{\text {bulk }}(z+d)$ with $V_{\text {bulk }}$ the potential at distance $z$ from a bulk. The scaling given by this simple argument is correct while the value of $C_{5}$ is not exact.

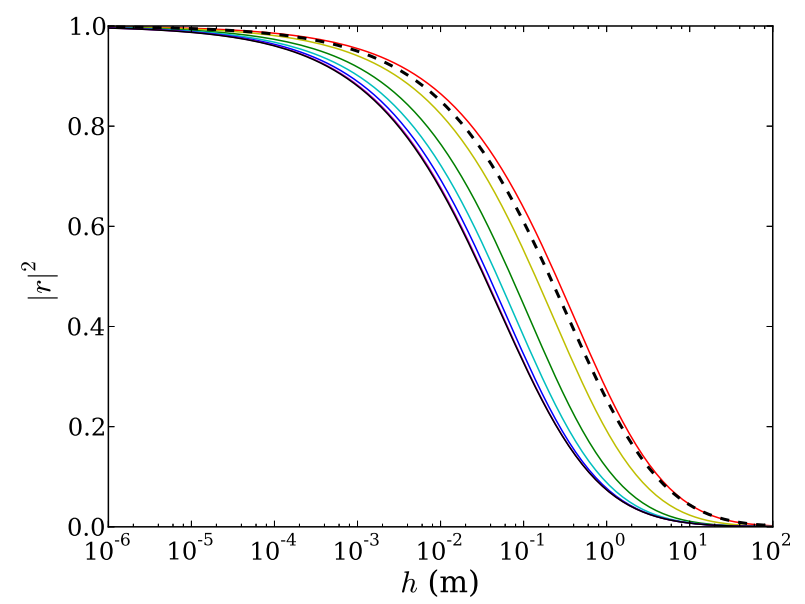

FIG. 5. (Color online) Quantum reflection probability $|r|^{2}$ as a function of the free fall height $h$ for $\overline{\mathrm{H}}$ atoms on silica slabs ; from bottom to top, the thickness is infinite (black), $50 \mathrm{~nm}$ (magenta), $20 \mathrm{~nm}$ (deep blue), $10 \mathrm{~nm}$ (light blue), $5 \mathrm{~nm}$ (deep green), $2 \mathrm{~nm}$ (light green) and $1 \mathrm{~nm}$ (red). The dashed line is the result for quantum reflection on non-doped graphene.

We depict in figure 5 the numerical solution for quantum reflection probability obtained with the exact $\mathrm{CP} / \mathrm{vdW}$ potentials for $\overline{\mathrm{H}}$ atoms falling to silica slabs with various values of the thickness (same color code as on figure 4). As expected, larger and larger values are obtained for the quantum reflection probability on thinner and thinner silica slabs, that is also steeper and steeper $\mathrm{CP} / \mathrm{vdW}$ potentials. For a free fall height $h \sim 10 \mathrm{~cm}$ for example, the probability $|r|^{2}$ reaches $\sim 50 \%$ for $3 \mathrm{~nm}$ slabs while it is only $33 \%$ pour Silica bulks. For comparison we also show the quantum reflection coefficient for graphene. Interestingly the same high quantum reflection than on a (not realistic) $1 \mathrm{~nm}$ slab can be obtained with the quantum reflection reaching $61 \%$ for non-doped graphene. This value increases only slightly $(\leq 2 \%)$ if doping is included.

\section{LOW ENERGY LIMIT}

We finally discuss the limit of near threshold quantum reflection, where the incident atomic energy $E$ goes to zero. Quantum reflection is thus characterized by a scattering length 23] which we will calculate in the present section for the different cases discussed above, with the aim of optimizing applications for manipulating antihydrogen with material walls [34, 35].

In order to conform to standard notation, we replace $p$ by $\hbar k$ in this section ( $k$ not to be confused with the 
electromagnetic wavevector used in the beginning of this paper). The reflection amplitude $r$ is a function of $k$ which can be written in terms of a complex-valued function $a(k)$ having the dimension of a length :

$$
r(k)=-\exp (-2 i k a(k))
$$

The real part of $a(k)$ determines the phase at reflection while its imaginary part determines the quantum reflection probability :

$$
|r|^{2}=\exp (4 k \operatorname{Im}(a(k))
$$

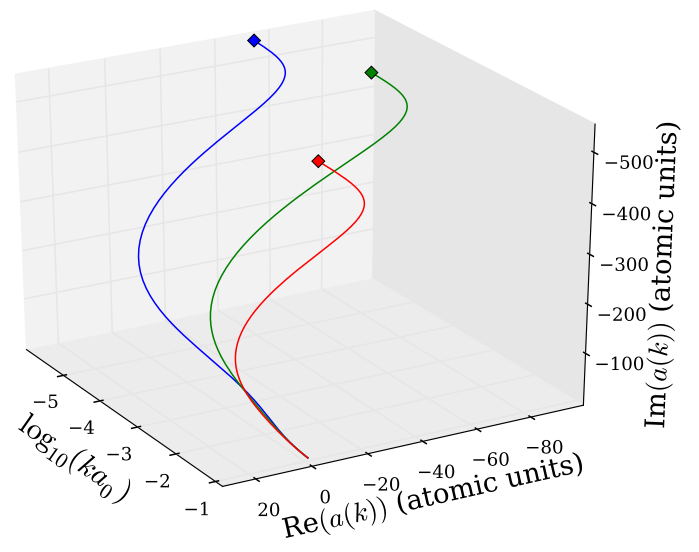

FIG. 6. (Color online) 3D representation of the variation of real and imaginary parts of $a(k)$ versus wavevector for $\overline{\mathrm{H}}$ atoms on bulk mirrors ; from top left to bottom right, perfect mirror (blue), silicon (green), silica (red).

We show in figure 6 the variations of real and imaginary parts of $a(k)$ versus wavevector (measured in atomic units) for $\overline{\mathrm{H}}$ atoms falling to perfect mirror, silicon and silica bulks (same color codes as in figure 2). We see that $a(k)$ goes to a finite value $a(0)$ when $k \rightarrow 0$, which is known as the scattering length; the values of $a(0)$ are collected in Table III

\begin{tabular}{|c|c|c|c|c|c|c|c|}
\hline \multicolumn{2}{|c|}{ perfect } & \multicolumn{2}{c|}{ silicon } & \multicolumn{2}{c|}{ silica } & \multicolumn{2}{c|}{ graphene } \\
\hline $\operatorname{Re}(a)$ & $\operatorname{Im}(a)$ & $\operatorname{Re}(a)$ & $\operatorname{Im}(a)$ & $\operatorname{Re}(a)$ & $\operatorname{Im}(a)$ & $\operatorname{Re}(a)$ & $\operatorname{Im}(a)$ \\
\hline-53.0 & -543.0 & -97.2 & -435.2 & -77.0 & -272.6 & -15.4 & -109.7 \\
\hline
\end{tabular}

TABLE III. Real and imaginary parts of the scattering length $a(0)$ for $\overline{\mathrm{H}}$ falling on perfect mirrors, silicon and silica bulks and graphene (given in atomic units $\mathrm{a}_{0}$ ).

We also show in figure 7 the variations of real and imaginary parts of $a(k)$ versus wavevector (measured in atomic units) for $\overline{\mathrm{H}}$ atoms on silica slabs (same color codes as in figure 5). Again, $a(k)$ goes to a finite value $a(0)$ when $k \rightarrow 0$, the real and imaginary parts of which are collected in Table IV

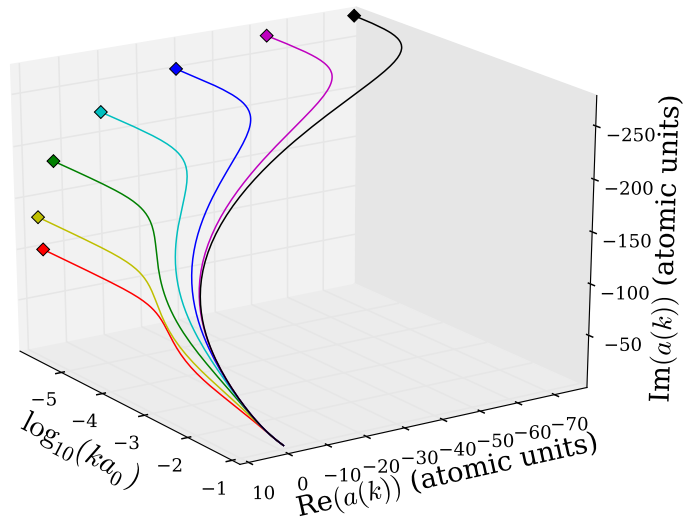

FIG. 7. (Color online) 3D representation of the variation of real and imaginary parts of $a(k)$ versus wavevector $\overline{\mathrm{H}}$ atoms falling to silica slabs ; from top right to bottom left, the thickness is infinite (black), $50 \mathrm{~nm}$ (magenta), $20 \mathrm{~nm}$ (deep blue), $10 \mathrm{~nm}$ (light blue), $5 \mathrm{~nm}$ (deep green), $2 \mathrm{~nm}$ (light green) and $1 \mathrm{~nm}$ (red).

\begin{tabular}{|c|c|c|c|c|}
\hline $\begin{array}{c}d \text { (slab } \\
\text { thickness) }\end{array}$ & \multicolumn{2}{|c|}{ silicon } & \multicolumn{2}{c|}{ silica } \\
\cline { 2 - 5 } & $\operatorname{Re}(a)$ & $\operatorname{Im}(a)$ & $\operatorname{Re}(a)$ & $\operatorname{Im}(a)$ \\
\hline $1 \mathrm{~nm}$ & 3.0 & -178.1 & 6.5 & -97.9 \\
$2 \mathrm{~nm}$ & 1.6 & -231.8 & 7.5 & -130.3 \\
$5 \mathrm{~nm}$ & -6.5 & -311.2 & 3.2 & -181.9 \\
$10 \mathrm{~nm}$ & -21.8 & -367.8 & -9.3 & -221.1 \\
$20 \mathrm{~nm}$ & -45.2 & -408.0 & -29.1 & -250.1 \\
$50 \mathrm{~nm}$ & -73.1 & -429.7 & -53.3 & -267.4 \\
$100 \mathrm{~nm}$ & -85.0 & -433.7 & -64.4 & -271.2 \\
bulk & -97.2 & -435.2 & -77.0 & -272.6 \\
\hline
\end{tabular}

TABLE IV. Real and imaginary parts of the scattering length of antihydrogen on silicon and silica slabs (given in atomic units $\left.\mathrm{a}_{0}\right)$.

We observe large variations of these values, which can have important applications for manipulating $\overline{\mathrm{H}}$ with material walls. By considering quantum gravitational traps for $\overline{\mathrm{H}}$ bounded below by the quantum reflection from the $\mathrm{CP} / \mathrm{vdW}$ potential and above by gravity, one obtains the following lifetime for the quantum bouncer in the first gravitational quantum state 34 :

$$
\tau=\frac{\hbar}{2 m g|\operatorname{Im} a(0)|}
$$

The lifetime is thus $\sim 5$ times larger for thin silica slabs than for the perfect mirrors considered in the calculations of 34. The same improvement holds for the width of resonances between quantum states which can be used for precise spectroscopic determination of the energies of these states, a technique which could allow a better accuracy for the gravitational behavior of $\overline{\mathrm{H}}$ atoms in future experiments [35]. 
The same techniques could also allow to trap antiatoms above curved material surfaces and them guide them at will during the longer lifetime achieved thanks to quantum reflection from steep potentials.

\section{CONCLUSION}

We have given realistic estimates of the $\mathrm{VdW} / \mathrm{CP}$ potential above matter slabs of arbitrary thickness and the corresponding reflection probability for antihydrogen atoms. It appeared that a substantial amount of quantum reflection is to be expected in the GBAR ex- periment. We gave a detailed analysis of the reflection process, solving the paradox of weaker potentials leading to higher reflection. Finally we have investigated the low-energy regime of quantum reflection and given quantitative predictions for the scattering length.

\section{ACKNOWLEDGEMENTS}

The authors thank the ESF Research Networking Programme CASIMIR (casimir-network.org) and the GBAR collaboration (gbar.in2p3.fr) for providing excellent possibilities for discussions and exchange.
[1] J. Lennard-Jones and A. Devonshire, Proc. Roy. Soc. London, A156, 6 (1936), A156, 29 (1936)

[2] H. Casimir and D. Polder, Nature, 158, 787 (1946) H. B. G. Casimir and D. Polder, Phys. Rev., 73, 360 (1948).

[3] V. U. Nayak, D. O. Edwards, and N. Masuhara, Phys. Rev. Lett., 50, 990 (1983).

[4] J. J. Berkhout, O. J. Luiten, I. D. Setija, T. W. Hijmans, T. Mizusaki, and J. T. M. Walraven, Phys. Rev. Lett., 63, 1689 (1989)

[5] I. Yu, J. Doyle, J. Sandberg, C. Cesar, D. Kleppner, and T. Greytak, Phys. Rev. Lett., 71, 1589 (1993).

[6] F. Shimizu, Phys. Rev. Lett., 86, 987 (2001)

[7] V. Druzhinina and M. DeKieviet, Phys. Rev. Lett., 91, $193202(2003)$

[8] H. Oberst, Y. Tashiro, K. Shimizu, and F. Shimizu, Phys. Rev., A71, 052901 (2005)

[9] V. P. A. Lonij, W. F. Holmgren, and A. D. Cronin, Phys. Rev. A, 80, 062904 (2009)

[10] B. S. Zhao, H. C. Schewe, G. Meijer, and W. Schoellkopf, Phys. Rev. Lett., 105, 133203 (2010)

[11] T. Pasquini, Y. Shin, C. Sanner, M. Saba, A. Schirotzek, D. Pritchard, and W. Ketterle, Phys. Rev. Lett., 93, 223201 (2004)

[12] T. A. Pasquini, M. Saba, G. B. Jo, Y. Shin, W. Ketterle, D. E. Pritchard, T. A. Savas, and N. Mulders, Phys. Rev. Lett., 97, 093201 (2006)

[13] M. Berry and K. Mount, Reports on Progress in Physics, 35, 315 (1972)

[14] W. Brenig, Zeitschrift für Physik B Condensed Matter, 36, 227 (1980), ISSN 0722-3277, 10.1007/BF01325286.

[15] D. Clougherty and W. Kohn, Phys. Rev., B46, 4921 (1992)

[16] C. Henkel, C. Westbrook, and A. Aspect, J. Opt. Soc. Am., B13, 233 (1996)

[17] C. Carraro and M. Cole, Prog. Surf. Sci., 57, 61 (1998)

[18] H. Friedrich, G. Jacoby, and C. Meister, Phys. Rev., A65, 032902 (2002).

[19] H. Friedrich and J. Trost, Physics Reports, 397, 359 (2004)

[20] T. E. Judd, R. G. Scott, A. M. Martin, B. Kaczmarek, and T. M. Fromhold, New Journal of Physics, 13, 083020 (2011)

[21] J. D. Perreault, M. Bhattacharya, V. P. A. Lonij, and A. D. Cronin, Phys. Rev. A, 77, 043406 (2008)
[22] V. Druzhinina, M. Mudrich, F. Arnecke, J. Madroñero, and A. Buchleitner, Phys. Rev. A, 82, 032714 (2010).

[23] A. Voronin and P. Froelich, Journal of Physics, B38, L301 (2005); A. Voronin, P. Froelich, and B. Zygelman, Phys. Rev., A72, 062903 (2005).

[24] Proposal to Measure the Gravitational Behavior of Antihydrogen at Rest (P. Pérez et al., 2011); see http: //gbar.in2p3.fr/public/SPSC-111025.pdf.

[25] G. Feinberg and J. Sucher, Phys. Rev., A2, 2395 (1970).

[26] M. Babiker and G. Barton, Journal of Physics A: Mathematical and General, 9, 129 (1976)

[27] J. M. Wylie and J. E. Sipe, Phys. Rev. A, 30, 1185 (1984)

[28] J. F. Babb, G. L. Klimchitskaya, and V. M. Mostepanenko, Phys. Rev. A, 70, 042901 (2004).

[29] K. A. Milton, Am. Journal of Physics, 79, 697 (2011)

[30] A. Lambrecht, P. A. Maia Neto, and S. Reynaud, New Journal of Physics, 8, 243 (2006)

[31] T. Emig, N. Graham, R. L. Jaffe, and M. Kardar, Phys. Rev. Lett., 99, 170403 (2007).

[32] R. Messina, D. A. R. Dalvit, P. A. Maia Neto, A. Lambrecht, and S. Reynaud, Phys. Rev., A80, 022119 (2009).

[33] A. Lambrecht, I. Pirozhenko, L. Duraffourg, and P. Andreucci, EPL, 77, 44006 (2007)

[34] A. Y. Voronin, P. Froelich, and V. V. Nesvizhevsky, Phys. Rev., A83, 032903 (2011).

[35] A. Y. Voronin, V. V. Nesvizhevsky, and S. Reynaud, Phys. Rev., A85, 014902 (2012) Journal of Physics, B45, 165007 (2012)

[36] Y. Zhang and D. P. Clougherty, Phys. Rev. Lett., 108, $173202(2012)$

[37] M. Marinescu, A. Dalgarno, and J. Babb, Phys. Rev., A55, 1530 (1997).

[38] C. Koughia, S. Kasap, and P. Capper, Springer Handbook of Electronic and Photonic Materials, Springer Handbook Of Series (Springer, 2006) ISBN 9780387260594.

[39] M. Bordag, I. V. Fialkovsky, D. M. Gitman, and D. V. Vassilevich, Phys. Rev. B, 80, 245406 (2009)

[40] Kummer's $M$ function is also known as the ${ }_{1} F_{1}$ hypergeometric function. It holds that $M\left(\frac{3}{2}, 4, z\right)=-16 L_{-3 / 2}^{(3)}(z)$ where $L_{n}^{(\alpha)}$ is a generalized Laguerre polynomial. More information can be found at http://dlmf.nist.gov. 
[41] S. Fulling and P. Davies, Proc. Roy. Soc. London, A348, 393 (1976)

[42] A. Lambrecht, M.-T. Jaekel, and S. Reynaud, European Physical Journal, D3, 95 (1998). 